\title{
Estrategias de aprendizaje emergentes en la modalidad e-learning
}

\author{
Learning Strategies Emerging in E-Learning Modality
}

\author{
Luis Demetrio Meza-López \\ Universidad Autónoma de Sinaloa (UAS). México. \\ luismezalopez@uas.edu.mx \\ Serafín Ángel Torres-Velandia \\ Universidad Autónoma del Estado de México (UAEM). México. \\ angelt@uaem.mx \\ José de Jesús Lara-Ruiz \\ Universidad Autónoma de Sinaloa (UAS). México. \\ jjlararuiz@gmail.com
}

\begin{abstract}
Resumen
El objetivo de esta investigación fue sistematizar las estrategias de aprendizaje que implementa el alumno de la modalidad e-learning. La problemática fue abordada desde una perspectiva cualitativa utilizando el método de la teoría fundamentada y la técnica de entrevista en profundidad. Se diseñó una plataforma tecnológica propia para realizar el trabajo de campo con entrevistas online, procesando los videos con un software de análisis cualitativo. La validez y confiabilidad de las categorías emergentes fue verificada mediante la saturación de los datos y trianguladas con un constructo teórico propio elaborado con referentes de Weinstein y Meyer (1998), Pintrich, García y Mckeachie (1991) y Tsai (2009). Los resultados muestran que hay una dimensión de estrategias de aprendizaje en la modalidad e-learning en donde el factor de gestión tiene gran influencia para regular el proceso sobre todo en el uso del tiempo y la administración de las estrategias. El factor de autorregulación considerando la metacognición y la adaptabilidad juegan un papel esencial, al igual que el factor sicológico de la motivación y la concentración, aunado al factor de usos de la tecnología en la selección y utilización de los medios tecnológicos. El aporte devela la forma con la que se adaptan estrategias emergentes de aprendizaje en la educación virtual en México y la posibilidad de que se conviertan en líneas de investigación para el contexto de América Latina.
\end{abstract}

\section{Palabras clave}

Estrategias de aprendizaje, modalidad e-learning, gestión, usos de la tecnología.

\begin{abstract}
The purpose of this investigation was the systematization of specific strategies that the student in e-learning modality uses for his learning. The problem was focused from a qualitative perspective supported on the method of grounded theory and the in-depth interview technique. An ad hoc technological platform waas designed to do fieldwork with online interviews, by processing videos with a qualitative analysis software. The validity and reliability of the result was triangulated with the theoretical construction made by the contribution of Weinstein and Meyer (1998), Pintrich, García and Mckeachie (1991) and Tsai (2009). These results show that there is a dimension of learning strategies in $e$ learning modality where the management factor has great influence to regulate the process, especially in the use of time and strategies administration. In addition, the self-regulation of the metacognition and the adaptability play an essential role, the same as the psychological factor of the motivation and concentration, combined with the factor of uses of the
\end{abstract}


technology for selecting and use the pertinent technologies on the adequate moment with the aim of learning. The contribution shows how learning strategies are adapted in virtual education in México and the possibility that they become lines of research for the Latin American context.

Key words

Induction, beginning teacher, b-learning, mentoring, learning community.

\section{Construcción de la problemática}

La problemática se inscribe en la consideración de que si en la modalidad presencial las estrategias de aprendizaje son categorizadas para su análisis y comprensión, en la modalidad a distancia/virtual también se presentan formas particulares de aprender con estrategias específicas a ese medio, las cuales si son develadas pueden categorizarse en un conocimiento sistemático.

Dado que en la modalidad presencial es común que el alumno presente un déficit en el uso de estrategias de aprendizaje (Pérez, Rodríguez, Borda \& Del Rio, 2003) luego entonces tal situación se complejiza en la modalidad virtual debido a la menor presencia de las interacciones cara a cara (face to face). Más aun, habría que asumir una ruptura epistemológica de una perspectiva lineal que intente transferir mecánicamente las estrategias de aprendizaje presenciales al entorno de los ambientes de aprendizaje virtuales.

Otro aspecto a tomar en cuenta son las diferencias entre el contexto americano y el europeo. Para Zambrano y Medina (2010), el desarrollo de la educación virtual en América, en comparación con Europa, se ha dado más lentamente, aunque existen universidades canadienses y estadunidenses que están asumiendo un papel importante, en el caso de Latinoamérica destaca, desde 1996, el modelo pedagógico virtual del Instituto Tecnológico de Estudios Superiores de Monterrey, por su parte Chile ha ido pionero en Sudamérica en modelos pedagógicos virtuales apoyados en el uso de computadoras y redes, en Argentina varias universidades se apoyan en las Tecnologías de la Información y la Comunicación (TIC) para desarrollar estrategias didácticas en distintos ámbitos educativos y productivos, en Brasil varias instituciones de educación superior basan su formación en el uso adecuado y creativo de las nuevas tecnologías con las exigencias empresariales del país. Algo similar sucede en Colombia donde se apoyan en plataformas tecnológicas y desarrollan proyectos de educación virtual. Esto nos lleva a considerar que el estudio de las estrategias de aprendizaje en la modalidad virtual tendrá diferencias en el caso mexicano y latinoamericano con el europeo.

Una forma de abordar la problemática es la evaluación de las estrategias de aprendizaje como un referente para comprender la eficacia de las mismas. En este proceso resulta de interés identificar coincidencias y discrepancias en las estrategias de aprendizaje usadas desde lo presencial y las estrategias de aprendizaje a distancia/virtual. El propósito de esta investigación fue contrastar las categorías emergentes o implícitas, utilizadas en la formación a distancia/virtual, con el conocimiento explícito de la temática, y sistematizar su diferenciación teniendo en todo momento el contexto propio de la modalidad e-learning, sobre todo en el caso de nuestro país que tiene similitudes con el resto de América Latina. 
Para el enfoque general se retomó el inventario de estrategias de estudio y aprendizaje LASSI (Learning and Study Strategies Inventory) de Weinstein y Meyer (1998) (aplicado en la modalidad presencial en más de 2700 universidades o instituciones en el mundo), también el aporte de Estrategias de Aprendizaje en Línea OLSS(Online Learning Strategies Scale) de Tsai (2009), y otros trabajos del campo temático lo que permitió identificar con mayor claridad diversas estrategias de aprendizaje, desde el plano conceptual, en ambas modalidades.

De los precedentes anteriores, se derivó que tanto en la modalidad presencial como en la modalidad a distancia/virtual hay estrategias de aprendizaje que tienen rasgos de coincidencia desde el punto de vista formal, pero como los medios son diferentes existen rasgos de diferenciación entre ambas modalidades. Por lo tanto se justificó la interrogante central ¿Cuáles son las estrategias de aprendizaje emergentes que el alumno utiliza o genera en la modalidad e-learning? Dicha interrogante marcó la pauta para el objetivo general sistematizar (identificar, analizar, categorizar y relacionar) las estrategias de aprendizaje emergentes que el alumno emplea en la modalidad. Precisando la importancia de que la respuesta a la pregunta central tenga lugar en un contexto in situ, es decir en la modalidad a distancia/virtual.

\section{Fundamentación teórica}

La educación a distancia en su evolución ha pasado por varios estadios o etapas las cuales van desde la correspondencia vía terrestre hasta la virtualidad, en esta última se utilizan los distintos aportes proporcionados por las TIC para soportar el proceso educativo en su totalidad, es el caso que Urban y Weggen (2000) en Marcelo, Ballesteros y Palazón (2002: 23) definen el e-learning como "el desarrollo de contenidos a través de cualquier medio electrónico, incluyendo internet, intranet, extranet, satélites, cintas de audio/video, televisión interactiva y $C D-R O M$ ', pudiendo observarse cierta tendencia tecnocéntrica. Por su parte Khan (2001) en Boneu (2007) desde una noción más pedagógica sostiene que el e-learning debe contener un diseño instruccional, pedagógico, tecnológico, de interfaz, evaluación, gerencia (administrador), soporte y ética de uso; es decir se trata de la mixtura de los recursos, la interactividad, el apoyo y actividades de aprendizaje estructuradas.

De manera integral Gros (2011) plantea que el e-learning ha pasado por tres generaciones, La primera generación la integra el modelo centrado en los materiales, es la adaptación de los materiales textuales a formatos web. Una segunda generación la integra el modelo centrado en el aula virtual, este comprende los Entornos Virtuales de Aprendizaje (EVA) y todo lo relacionado a estos. La tercera generación es la del modelo centrado en la flexibilidad y la participación que apoya la colaboración, las comunidades de aprendizaje en línea, contenidos especializados en línea y también los generados por los estudiantes, así como el uso de cualquier tecnología que los favorezca; donde los alumnos son conscientes de su propio aprendizaje y de como lograrlo. En el caso de México y América Latina, en general, no se ha logrado alcanzar el nivel de la última generación de e-learning ya que esto depende de situaciones muy particulares o especificas a cada país, como lo son, por mencionar algunas, las de tipo económico, tecnológicas, sociales y educacionales, como sostiene Schwartzmann (2001) en Arrollo y Milkos (2008) al señalar que difícilmente se logrará superar el

Estrategias de aprendizaje emergentes en la modalidad e-learning. Luis Demetrio Meza-López, Ángel Serafín Torres-Velandia y José de Jesús Lara-Ruiz.

Página 3 de 21 
abismo científico-tecnológico o las brechas sociales en América Latina y por ende no se alcanzarán los niveles de desarrollo y madurez educacional requeridos para conseguir las metas de cobertura y calidad de la región.

Ahora bien las diferentes definiciones que se han dado de estrategias cognitivas y/o estrategias de aprendizaje coinciden sustancialmente en que son un conjunto de procedimientos o procesos mentales empleados por una persona en una situación particular de aprendizaje para facilitar la adquisición de conocimientos (Derry \& Murphy, 1986). Al respecto se considera significativo que el alumno tome conciencia de su forma de aprender y que sea proclive a la autorregulación de sus acciones para optimizar su aprendizaje. De la temática existen múltiples tipologías o taxonomías por lo que a continuación se presentan las más relevantes:

Existen estrategias de aprendizaje que se definen por las dicotomías: 1) consciente (controlada)-inconsciente (automática); 2) autodirigida (individual y espontanea)heterodirigida (interactiva y mediada por la instrucción); y 3 ) genérica (global, utilizable en cualquier situación de aprendizaje)-específica (aplicable a un dominio, campo o tema restringido, a la que a veces se le denomina táctica) (Mayor, Suengas \& Marqués, 1995). Algunos autores admiten la existencia de estrategias en cada uno de estos polos, considerándolas como dicotómicas y opuestas o bien como polos de un continuum, en ocasiones se han excluido o minimizado algunas de estas estrategias pero, en cualquier caso, se tiende a concebirlas como conscientes, autodirigidas y genéricas.

Por su parte, Weinstein y Mayer (1986) clasifican las estrategias en:

- Estrategias de ensayo (básicas y complejas).

- Estrategias de elaboración (básicas y complejas).

- Estrategias de organización (básicas y complejas).

- Estrategias de revisión y evaluación de la comprensión.

- $\quad$ Estrategias afectivas y motivacionales.

Pintrich et al. (1991) plantea que pueden distinguirse tres grandes tipos de estrategias de aprendizaje:

- Estrategias cognitivas: son las estrategias de repaso, elaboración y organización de la información, además del pensamiento crítico.

- Estrategias metacognitivas: consisten en la planificación, control y regulación de las actividades realizadas durante el aprendizaje.

- Estrategias de regulación de recursos: Comprenden la organización del tiempo y el ambiente de estudio, regulación del esfuerzo, aprendizaje con pares y búsqueda de ayuda.

En esta investigación se realizó una búsqueda exhaustiva de instrumentos que evalúan o tratan las estrategias de aprendizaje, ${ }^{1}$ destacando el trabajo de Weinstein y Meyer

\footnotetext{
${ }^{1}$ Encontrando precedentes desde mediados de 1960 donde la mayoría aborda la temática desde la perspectiva presencial con excepción del OLSS que integra el aprendizaje en línea, asimismo LASSI for Learning Online (LLO), el cual no se incorpora debió a que surge después de esta indagatoria.
} 
(1998) el cual se concreta en el instrumento LASSI ${ }^{2}$ que consta de 80 ítems los cuales están distribuidos en diez escalas para su evaluación: 1) Ansiedad: esta escala evalúa el grado en que los estudiantes se preocupan por la escuela y su rendimiento académico; 2) Actitud: en este apartado se evalúa las actitudes de los estudiantes y el interés en la escuela, así como el éxito o logros académicos; 3) Concentración: se percibe la capacidad del alumno para dirigir y mantener la atención en las tareas; 4) Motivación: esta escala evalúa la diligencia de los estudiantes, la autodisciplina, y la voluntad de hacer el esfuerzo necesario para completar con éxito los requisitos académicos; 5) Selección de Ideas Principales: aprecia la habilidad del estudiante en relación a si logra catalogarla información importante de la menos importante para su aprendizaje; 6) Procesamiento de la información: evalúa si el estudiante puede usar las imágenes, la elaboración verbal, estrategias de organización, y habilidades de razonamiento, como el aprendizaje de estrategias para ayudar a construir puentes entre lo que ya saben y lo que están tratando de aprender y recordar, es decir, la adquisición de conocimientos, la retención y la aplicación en el futuro; 7) Auto-Prueba: valora si el estudiante hace uso de la revisión y comprensión de técnicas de vigilancia para determinar su nivel de comprensión de la información que tiene que aprender; 8) Estudio de la ayuda: se encarga de evaluar el uso que el estudiante hace con los apoyos o recursos que están disponibles para ayudarles a aprender o retener información; 9) Gestión del Tiempo: esta escala evalúa la administración que el alumno hace para regular su tiempo en relación a sus actividades académicas, y 10) Probar las estrategias: encargada de evalúar como los estudiantes se preparan para ensayar las estrategias que tienen que tomar en situaciones determinadas.

Las anteriores escalas son aplicadas sólo para la modalidad presencial, para la modalidad orientada al aprendizaje en línea se tiene el estudio Online Learning Strategies Scale (OLSS) de Tsai, Meng-Jung (2009),el cual consta de 20 ítems, contenidos en cinco escalas para su medición: Autocontrol, Motivación, Concentración, Ansiedad en el internet e Información de internet.

La presente investigación tomó en cuenta el paradigma sociocultural y cognitivo, los trabajos mencionados de Pintrich y García (1993), Pintrich et al. (1991), los planteamientos de Weinstein y Meyer (1998) concretados en el instrumento LASSI, y el aporte OLSS de Tsai (2009). Los referentes teóricos de los autores citados, se contrastaron y/o relacionaron con las estrategias de aprendizaje utilizadas por alumnos de la modalidad e-learning, resultando en una primera aproximación teórica-empírica de las dimensiones: a) Usos de recursos (gestión del tiempo, estudio de la ayuda); b) Cognitiva (procesamiento de la información en internet, selección de ideas); c) Metacognitiva (autocontrol y probar las estrategias), y d) la dimensión Factores sicológicos (motivación, ansiedad, actitud y concentración).(Ver figura 1).

\footnotetext{
${ }^{2}$ El catálogo de LASSI para internet es de reciente difusión, por lo cual incorporarlo al presente estudio amerita la continuidad de la investigación, por el momento se considera que el artículo cubre los requisitos de publicación de la revista. LASSI presenta distintas versiones que se pueden consultar en: $<$ http://www.hhpublishing.com/>
}

Estrategias de aprendizaje emergentes en la modalidad e-learning. Luis Demetrio Meza-López, Ángel Serafín Torres-Velandia y José de Jesús Lara-Ruiz. 
Figura 1. Dimensiones de las estrategias de aprendizaje relacionadas con las escalas de LASSI-OLSS. (Constructo teórico) ${ }^{3}$.

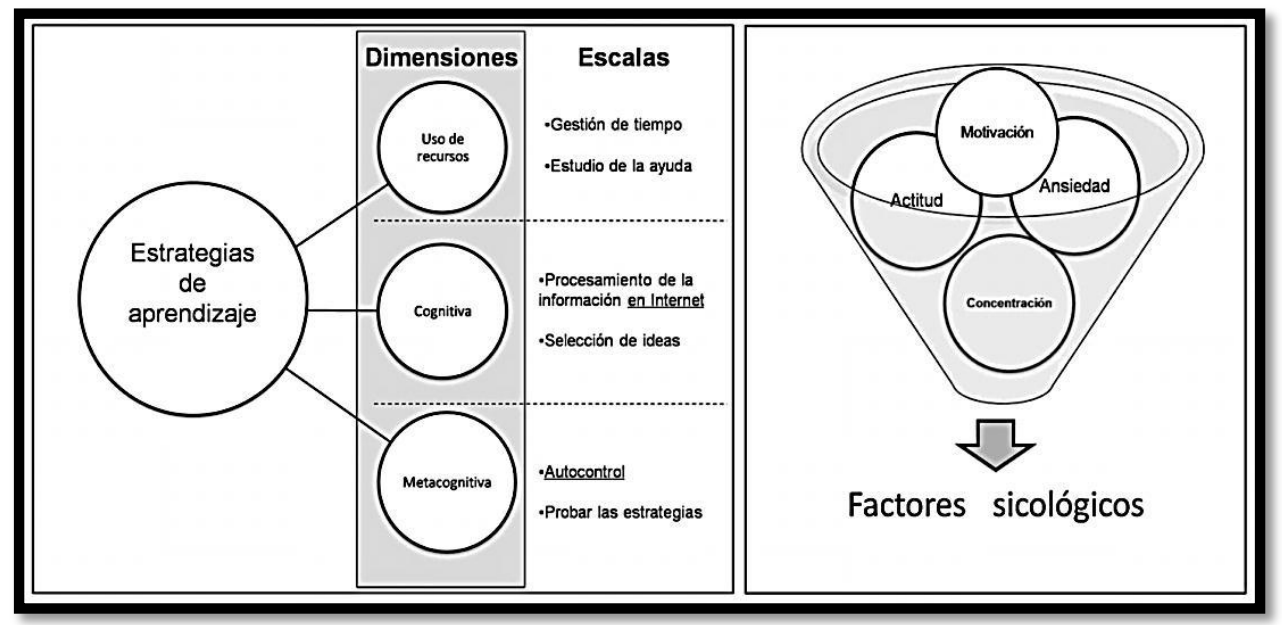

Fuente: elaboración propia contrastada y/o relacionada con las escalas LASSI de Weinstein y Meyer (1998); Pintrich y García (1993); Pintrich et al. (1991), y OLSS de Tsai (2009).

Dicho constructo teórico-empírico de dimensiones observado desde la tipología de Pintrich et al.(1991) pero también desde el aprendizaje online, como fue señalado, muestra claramente que emanan categorías que requerían una mayor profundización como son: la gestión del tiempo y los tipos de ayuda en la plataforma virtual, las formas de procesar la información en internet, la selección de ideas propias por parte del alumno, el autocontrol en el proceso de aprendizaje en línea, además de la vigilancia de las estrategias implementadas. Lo anterior, sin dejar de lado aspectos subjetivos de tipo motivacional, y desde luego las tensiones que se presentan entre las actitudes, la concentración y la ansiedad generadas en el entorno del e-learning.

En general visto el proceso educativo como un todo, independientemente del tipo de modalidad, se dan aspectos que son comunes, hayestudiantes que son capaces no sólo de aprender, sino de mejorar esas capacidades adquiriendo estrategias para autoregular su propio aprendizaje; igualmente los hay capaces, no sólo de pensar, sino de aprender a pensar mejor utilizando estrategias cognitivas cuya selección y aplicación autocontrolan: se trata de sujetos que han adquirido y desarrollado habilidades metacognitivas que pueden aplicar al aprendizaje o al pensamiento (Mayor et al.,1995).

Al respecto, es relevante destacar el trabajo de Collis y Meeuwsen (1999) quienes ya advertían las principales diferencias del aprendizaje en los estudiantes que hacen uso del internet. Ellos consideran los tipos de habilidades y enfoques de aprendizaje en línea que necesitan los estudiantes para hacer empleo del potencial que ofrece el internet. En particular, describen los retos del entorno online con respecto a componentes tales como "aprender a aprender", incluyendo la articulación y la reflexión, técnicas de estudio, encontrar y aplicar ejemplos pertinentes, así como la autoevaluación, la búsqueda y selección de información significativa que está en internet y su integración al aprendizaje. Sin embargo Collis y Meeuwsen (1999) sostienen, que los estudiantes

\footnotetext{
${ }^{3}$ Precisando que de las diez escalas presentadas, en el primer recuadro cuatro se retoman de LASSI y dos, las subrayadas, corresponden a Tsai, la dimensiones plasmadas son la de la tipología de Pintrich et al. (1991). Por su parte las cuatro escalas de factores sicológicos son de Weinstein y Meyer (1998).
}

Estrategias de aprendizaje emergentes en la modalidad e-learning. Luis Demetrio Meza-López, Ángel Serafín Torres-Velandia y José de Jesús Lara-Ruiz. 
pueden no estar familiarizados con aspectos tecnológicos de los entornos basados en internet y como resultado de este "nuevo" modelo de aprendizaje, puede ser necesario perfilarlas estrategias que usan en ambientes de aprendizaje en línea.

\section{Aspectos metodológicos y técnicos}

El enfoque utilizado en la investigación fue de corte cualitativo considerando la perspectiva epistemológica del construccionismo, es decir centrando la atención en un mundo de intersubjetividad compartida (Berger \& Luckmann, 1993). La perspectiva teórica fue el interpretativismo sustentado en el interaccionismo simbólico (Blumer, 1969 \& Mead, 1964), con el método de la teoría fundamentada (Glaser \& Strauss, 1967 y Strauss \& Corbin, 2002) que surge de los datos y no a la inversa. Se parte de una teoría mínima (en este caso desde los planteamientos de Weinstein et al. (1998), Pintrich et al. (1991) y Tsai (2009).

En efecto no corresponde con el estado del arte del e-learning pero se considera que cubre los requisitos mínimos de acuerdo con el método implementado. Como informantes participaron 5 (cinco) ${ }^{4}$ estudiantes de diversos estados de las República Mexicana (fueron seleccionados con base a los criterios de estudiantes sobresalientes, regulares y de nivel apenas suficiente desde su desempeño académico), quienes fueron entrevistados dos veces en dos etapas de la investigación, y de acuerdo con el método de la teoría fundamentada se identificaron las categorías emergentes y los datos correspondientes hasta llegar a su saturación.

Desde la perspectiva cualitativa no se exige una muestra probabilística representativa sino informantes de calidad a quienes se entrevista a profundidad mediante el método de comparación constante hasta que los participantes han cubierto su aporte en la construcción de los conceptos, categorías y dimensiones identificadas por ellos mismos. Respecto a la no participación de alumnos de otras áreas del conocimiento atiende a una delimitación de la muestra de estudio a juicio de los investigadores. De manera que queden abiertas para América Latina líneas de investigación a ser abordadas en diferentes contextos y campos disciplinares.

Las técnicas de procedimientos de recogida y análisis de datos fueron entrevistas en profundidad para comprender las perspectivas y experiencias de los informantes en la dimensión subjetiva estrategias de aprendizaje en la modalidad e-learning.

En relación a los participantes en esta investigación fueron identificados sus testimonios como $A, B, C, D$ y $F$ a fin de preservar su anonimato. Sólo se menciona que son alumnos de un Doctorado en Tecnología Educativa cursando la modalidad virtual, de una Institución Particular de Educación Superior (con Registro de Validez Oficial de la Secretaría de Educación Pública), que radican en distintos estados de México.

Para realizar el trabajo de campo, primeramente se vio la necesidad de desarrollar un instrumento tecnológico denominado Sistema Digital para Entrevistas Interactivas Vía

\footnotetext{
${ }^{4}$ Referente al número de informantes se coincide con lo planteado por Patton (1990) en Robles (2011), acerca de que resulta complicado determinar un número mínimo o máximo de entrevistados, pues la finalidad no obedece a una representación estadística, sino que consiste en el estudio minucioso de la información que se obtenga de las conversaciones con los entrevistados.
}

Estrategias de aprendizaje emergentes en la modalidad e-learning. Luis Demetrio Meza-López, Ángel Serafín Torres-Velandia y José de Jesús Lara-Ruiz. 
Web $\left(\right.$ SDEIVW) ${ }^{5}$ el cual sirvió de soporte en las entrevistas online. A través del SDEIVW se llevaron a cabo entrevistas mediadas de manera sincrónica "cara a cara" con cada uno de los participantes, sin necesidad de coincidir geográficamente, y desde la comodidad donde se encontraban los informantes; lo que se tradujo en optimización del aspecto económico y del tiempo requerido para el estudio ${ }^{6}$. Las entrevistas online son una forma de indagar ad hoc al medio virtual en el cual el estudiante se está formando, con lo que se logra captarlo que dice y sus gestos, gracias a la observación e interacción de su lenguaje corporal (quedando registrado el evento de manera digital).

La acción de realizar entrevistas en profundidad es un arte complejo y más si las interacciones se dan en la virtualidad como es el caso que acontece. Por ello, antes de hacer cualquier contacto con los informantes se elaboró una guía o formato como lo requiere la técnica en esta investigación (Robles, 2011) (ver Cuadro 1), donde se plasmaron los contenidos o temáticas a tratar así como los momentos de la misma.

Cuadro 1. Guía flexible I para entrevista online

\begin{tabular}{|c|c|c|}
\hline Fase & Lineamientos generales a indagar & $\begin{array}{l}\text { Conceptos } \\
\text { relacionados } \\
\text { con el de } \\
\text { "estrategia" }\end{array}$ \\
\hline Inicio & $\begin{array}{l}\text { - Dar la bienvenida. } \\
\text { - Exponer los lineamentos de la entrevista online } \\
\text { - } \quad \text { Romper el "hielo" con comentarios de orden general }\end{array}$ & \multirow{3}{*}{$\begin{array}{l}\text { Acción } \\
\text { Táctica } \\
\text { Organizar } \\
\text { Planear } \\
\text { Objetivos } \\
\text { Proceso } \\
\text { Operaciones } \\
\text { Maniobra } \\
\text { Habilidad } \\
\text { Destreza } \\
\text { Pericia } \\
\text { Apoyos } \\
\text { Competencia }\end{array}$} \\
\hline Intermedio & $\begin{array}{l}\text { - } \quad \text { Empezar con la formalidad: Propiciar que el entrevistado } \\
\text { comente sobre cómo fue, o es, su formación como alumno en } \\
\text { la virtualidad. } \\
\text { - } \quad \text { Dispositivos de conexión. } \\
\text { - } \quad \text { Pateriales didácticos utilizados (cuestionar iaprende?). } \\
\text { columna 3). } \\
\text { - } \quad \text { Estrategias para aprender en la virtualidad. } \\
\text { - } \quad \text { Adrabajo en equipo para realizar las tareas. } \\
\text { - La modalidad e-learning como estrategia. }\end{array}$ & \\
\hline \multirow[t]{2}{*}{ Cierre } & $\begin{array}{l}\text { - Tiene algo que agregar. } \\
\text { - } \quad \text { Pactar otra entrevista de ser necesario. } \\
\text { - } \quad \text { Despedida. }\end{array}$ & \\
\hline & Observaciones / anotaciones & Fecha de entrevista \\
\hline
\end{tabular}

Fuente: elaboración propia.

Dicha guía contempló los aspectos: 1) la "Fase"; 2) "Los lineamientos generales a indagar", y 3) "Conceptos relacionados con el de estrategia". En el proceso de entrevista se conjugó la "Fase" con los "Lineamientos generales a realizar", resultando tres lapsos o tiempos:

a) El primero llamado de "inicio" donde se da la bienvenida al entrevistado, se exponen los lineamientos de la entrevista online y se empieza establecer rapport con el entrevistado.

\footnotetext{
${ }^{5}$ El SDEIVW estaba contenido en el dominio Web <http://www.iveame.mx >

${ }^{6}$ En contraste con las entrevistas convencionales (presenciales) las cuales requieren que el infórmate y el entrevistado se desplacen a un punto de encuentro.
}

Estrategias de aprendizaje emergentes en la modalidad e-learning. Luis Demetrio Meza-López, Ángel Serafín Torres-Velandia y José de Jesús Lara-Ruiz. 
b) El nombrado "Intermedio" se centra en ir abordando la temática a tratar, de lo general a lo particular, incluyendo los cuestionamientos de manera gradual y ordenada. Donde el mismo informante diría si es una estrategia que concibe para aprender ya que para el entrevistador es un término, o palabra reservada, que se utiliza ya que el entrevistado la emplea.

c) El denominado "Cierre" donde se pregunta al entrevistado si tiene algún comentario complementario y, así mismo, de requerirse, ver la posibilidad de pactar una nueva entrevista online. Cubiertos esos puntos se procede a la despedida que incluye el agradecimiento por participar.

Los datos fueron analizados con el Método Comparativo Constante (MCC) de la teoría fundamentada el cual consiste en la comparación constante de similitudes y diferencias, de las incidencias identificadas en los datos con el objetivo de descubrir patrones de comportamiento que se repitan. Este método de análisis, contribuye al desarrollo de una teoría fundamentada en los datos, gracias a que el investigador refina esos conceptos, identifica sus propiedades, explora sus interrelaciones y los integra en una teoría coherente, como sustentan Glaser y Strauss (1967); al mismo tiempo que se codifica, se analiza para elaborar conceptos, mediante comparaciones constantes de las incidencias específicas de los datos. Por lo anterior y no habiendo llegado a una saturación del dato después de realizar la sistematización del análisis de los mismos con la teoría fundamentada y develar categorías emergentes, se procedió a realizar una segunda ronda de entrevistas online (ver Cuadro 2) en el SDEIVW donde se aborda la temática tocando puntos específicos con cada uno de los informantes.

Cuadro 2. Guía flexible II para entrevista Online

\begin{tabular}{|c|c|c|}
\hline $\begin{array}{l}\text { Categoría } \\
\text { emergente }\end{array}$ & PUNTOS A TRATAR & Subcategoría \\
\hline \multirow{2}{*}{ 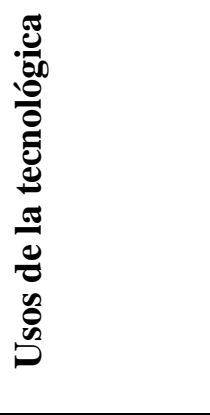 } & $\begin{array}{l}\text { Opinión sobre: conocer la gama tecnológica } \\
\text { que puede emplear el alumno en la modalidad } \\
\text { (ejemplifique) }\end{array}$ & $\begin{array}{c}\text { Seleccionar de } \\
\text { forma inteligente las } \\
\text { alternativas } \\
\text { tecnológicas } \\
\end{array}$ \\
\hline & $\begin{array}{l}\text { O Relevancia del momento de emplear la } \\
\text { tecnología. }\end{array}$ & $\begin{array}{c}\text { Determinar el } \\
\text { momento de su } \\
\text { utilización }\end{array}$ \\
\hline \multirow{2}{*}{ 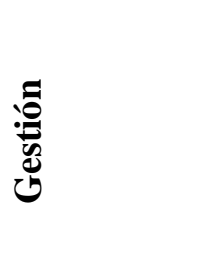 } & $\begin{array}{l}\text { El tiempo como factor determinante para el } \\
\text { funcionamiento de las Estrategias de } \\
\text { aprendizaje en la modalidad e-learning. }\end{array}$ & Tiempo \\
\hline & $\begin{array}{l}\text { - Se cuenta con un orden a la hora de aplicar las } \\
\text { estrategias de aprendizaje. }\end{array}$ & $\begin{array}{l}\text { Control de las } \\
\text { estrategias }\end{array}$ \\
\hline
\end{tabular}

Estrategias de aprendizaje emergentes en la modalidad e-learning. Luis Demetrio Meza-López, Ángel Serafín Torres-Velandia y José de Jesús Lara-Ruiz. 


\begin{tabular}{|c|c|c|c|}
\hline \multirow{2}{*}{ 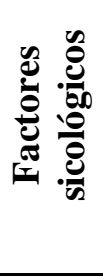 } & & $\begin{array}{l}\text { El estar enfocado/concentrado, ¿cómo } \\
\text { considera que impacta en el aprendizaje? }\end{array}$ & \multirow{2}{*}{$\begin{array}{c}\text { Concentración } \\
\text { Motivación }\end{array}$} \\
\hline & & $\begin{array}{l}\text { El sentirse motivado en la modalidad } \\
\text { e-learning, cómo lo considera, ¿es relevante? }\end{array}$ & \\
\hline \multirow{2}{*}{ 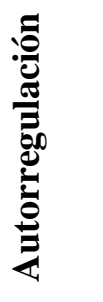 } & 0 & $\begin{array}{l}\text { Comprensión del alumno de manera macro } \\
\text { (general) y micro (particular) de cómo es que } \\
\text { aprende. }\end{array}$ & Metacognición \\
\hline & & $\begin{array}{l}\text { Que hace el alumno ante los cambios del medio } \\
\text { educativo ó ante los requisitos que le solicitan. }\end{array}$ & Adaptabilidad \\
\hline
\end{tabular}

Fuente: elaboración propia

De manera general, los datos recolectados de las estrategias de aprendizaje en la modalidad e-learning fueron analizados en cuatro momentos o fases: El primero fue recolectar los datos, esta parte la integra las videograbaciones de las entrevistas online; el segundo consistió en organizar los datos, lo que se realizó mediante el programa informático para el análisis de datos cualitativos Atlas.ti (ver.7.5); el tercero fue el análisis de los datos ${ }^{7}$, utilizando el método comparativo constante, con su codificación abierta, axial y selectiva para su tratamiento, y finalmente el desarrollo de teoría, la que fue producto de la saturación teórica en la etapa anterior. En lafigura2, se sintetiza lo expuesto y brinda un panorama general de cómo se procedió en esta investigación para la obtención de las categorías emergentes (teoría sustantiva).

Figura 2. Sistematización del análisis de los datos con la teoría fundamentada.

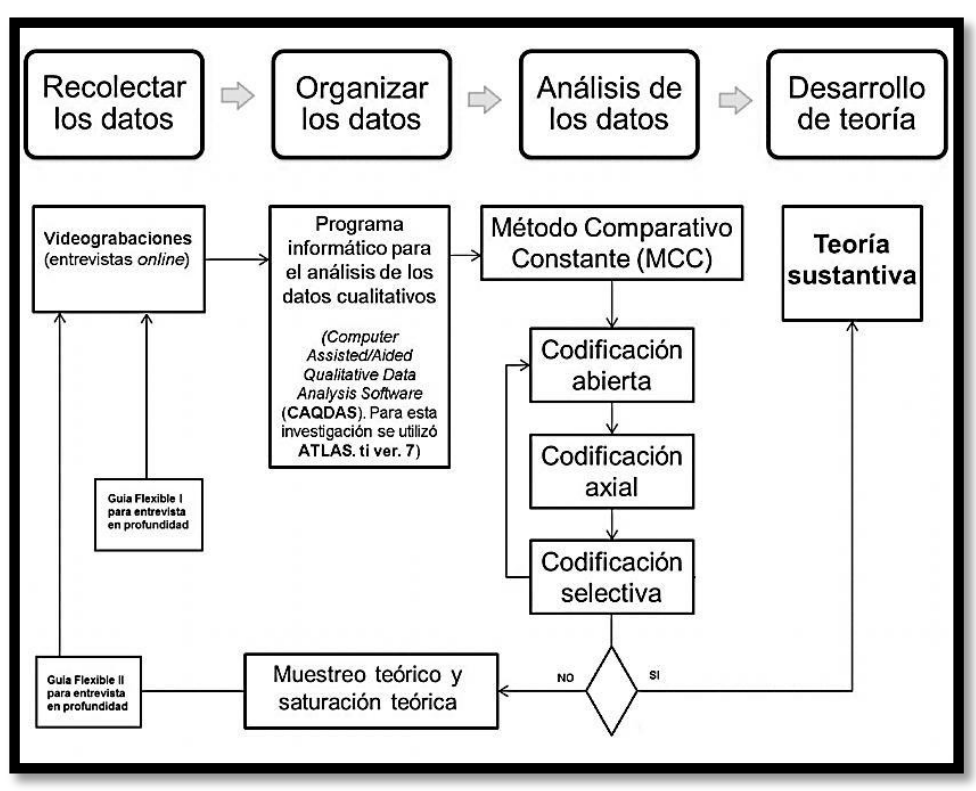

Fuente: elaboración propia.

\footnotetext{
${ }^{7}$ Las videograbaciones se procesaron de manera directa con el software Atlas.ti, lo cual es una manera novedosa y práctica para su tratamiento. Una de las ventajas que ofrece el hacer el análisis de manera directa a la videograbación específicamente en el formato denominado AVI (Audio Video Interleave), es que no hay que transcribir la videograbación lo cual se traduce en una reducción considerable de tiempo.
}

Estrategias de aprendizaje emergentes en la modalidad e-learning. Luis Demetrio Meza-López, Ángel Serafín Torres-Velandia y José de Jesús Lara-Ruiz. 
Es importante destacar que para efectos de validez en la investigación con el Atlas.ti se identificó el grado de saturación de los códigos, a través de la función code-primarydocuments-table (códigos-documentos primarios-tablas), que muestra la cantidad de citas que cada código presenta lo que es requerido para el método de la teoría fundamentada.

El Atlas.ti, según Flick, (2007), es un programa de recuperación de texto, sin embargo está dirigido a un trabajo conceptual, en donde cada paso de la codificación abierta, axial y selectiva tiene un espacio en el programa. Por ejemplo, la codificación abierta se realiza en el nivel de codificación de segmentos, ya sea de texto, audio, video (como es el caso) o imágenes. En tanto, la codificación axial se da con la función creación de redes de relaciones conceptuales. Weitzman y Miles (1995) sostienen que son esquemas explicativos, pero no jerárquicos, de las categorías y sus subcategorías en función de las relaciones entre familias de códigos o anotaciones. En cuanto, a la codificación selectiva propuesta en la teoría fundamentada, el Atlas.ti posee la función denomina súper código, que permite elaborar una categoría central que integra los códigos y categorías construidos en las fases de codificación abierta y axial. A decir de San Martín (2014) el uso del Atlas.ti facilita la organización del análisis a través de funciones que permiten principalmente: segmentar las citas, conceptualizar, registrar reflexiones, categorizar, relacionar procesos y mostrar la teoría que se construye a través de diagramas. En síntesis la utilización de la herramienta informática de análisis cualitativo Atlas.ti fue un gran apoyo para realizar procedimientos de primer y segundo orden, favoreciendo un mayor poder de comprensión del objeto de estudio.

\section{Resultados e interpretación}

Con el sustento de la teoría fundamentada (aplicando la codificación abierta del MCC), el interaccionismo simbólico (Blumer, 1969 \& Mead, 1964), además de los resultados de la primera ronda de entrevista online con los informantes, se develó en la segunda entrevista en profundidad la categoría núcleo "Las estrategias de aprendizaje en la modalidad e-learning", la cual está concatenada a cuatro categorías emergentes: a) Autorregulación; b) Factores sicológicos; c) Gestión, y d) Usos de la tecnología, en donde se encontró que a cada código le corresponden dos subcategorías.

La categoría Autorregulación se asocia a la Metacognición y Adaptabilidad; para la categoría Factores sicológicos son Concentración y la Motivación; en el código de Gestión se vincula con el Tiempo y Control de las estrategias, y la categoría Usos de la tecnología se liga a Seleccionar de forma inteligente las alternativas tecnológicas y Determinar el momento de su utilización.

En primera instancia se conceptualizan ${ }^{8}$ las categorías básicas, posteriormente se explican sus componentes y se incluye la información más destacada según el testimonio de los informantes:

\footnotetext{
${ }^{8}$ El sustento de los conceptos tienen su origen principal en el análisis de las entrevistas online y los autores que se presentaron en el marco teórico, destacando Weinstein et al. (1998) con su instrumento LASSI y OLSS de Tsai (2009).
}

Estrategias de aprendizaje emergentes en la modalidad e-learning. Luis Demetrio Meza-López, Ángel Serafín Torres-Velandia y José de Jesús Lara-Ruiz. 
Autorregulación: es la capacidad del estudiante para poder regular por sí mismo las acciones que sean necesarias para su aprendizaje. Destacando lo que comentan los informantes $A$ y $F$ :

[...] ser organizados con cada una de las actividades que se emprenden en la escuela lo cual es indispensable para poder aprender.

En su testimonio $C$ dice:

[...] que él decide que hacer primero o después [regular] para lograr el objetivo del aprendizaje.

- Metacognición: es la capacidad del alumno de autorregular sus procesos mentales para orientarlos al aprendizaje deseado, donde macroprocesos cognitivos o superiores gestionan procesos simples o elementales. $A$ expresa:

[...] tengo un panorama general como una especie de guía mental que me ayuda a hacer las tareas simples.

Con lo que coinciden $D$ y $F$.

- Adaptabilidad: es la relación que el alumno tiene con su entorno inmediato y que de manera consciente toma decisiones para enfrentar los requerimientos o exigencias que se le presentan. Por lo común son un producto causado por su entorno. $B$ expresó:

[...] yo me fijo en el maestro si viene muy light, pues yo me adapto conforme sea el maestro y sus exigencias.

Lo anterior hace alusión a que el docente no es muy exigente en su clase, siendo la finalidad salir avante en la asignatura online. Argumento similar sucede con $A, C$ y $D$.

Factores sicológicos: son aquellos que tienen que ver con cuestiones intrínsecas del individuo y que pueden propiciar el aprendizaje.

- Motivación: surge desde el interior del individuo es decir es intrínseca pero está supeditada a factores externos, como puede ser el mismo entorno virtual. $C$ comenta:

[...] que tienen muchos trabajos y que en ocasiones los maestros no ayudan [haciendo alusión a motivarlos (factor externo)] para que uno se sienta con ganas de realizar los ensayos.

Por su parte $B$ comenta que:

[...] hay días que, la verdad es que, uno tiene que hacer otras cosas y no dan ganas de hacer los trabajos aunque los hago, pero sin muchas ganas.

El estado anímico en un alumno es primordial (factor interno) pues es lo que impulsa a realizar más y mejor las actividades académicas. 
- Concentración: capacidad para poder enfocarse en las distintas tareas asignadas $A$, $B, \mathrm{C}, D$ y $F$, coinciden en que:

[...] es necesario enfocarse para que cualquier actividad académica salga bien, además es básico e indispensable para el aprendizaje.

Gestión: en la modalidad e-learning es un factor determinante para que el alumno pueda aprender, pues es indispensable poder regular y/o controlar lo que acontece en la misma. La relevancia de la gestión según $A, B, C, D$ y $F$ es hacer posible:

[...] seguir con los estudios sin descuidar el trabajo o la familia, lo que con otra modalidad no podría.

Conviene destacar, que la categoría emergente de Gestión se asocia y/o tiene que ver con la autogestión del alumno y que según Milkos y Arroyo (2008) es de gran relevancia en un escenario de prospectiva de la educación a distancia y del e-learning en América Latina, al considerar la autogestión como el proceso de reconocimiento, adaptación, decisión y acción en los propios procesos de aprendizaje, sean estos institucionales o individuales. De esta manera la gestión o autogestión vendrían siendo una línea rectora en las estrategias de aprendizaje emergente en el modelo pedagógico del e-learning.

- Tiempo: se puede clasificar en sincrónico y asincrónico, en el primero se requiere que el alumno este al unísono (en tiempo real) con lo que se le solicita en la escuela, en el segundo, lo anterior no es necesario puesto que el alumno puede hacerlo en cualquier momento (en el horario que más le convenga), $A, B, C, D$ y $F$, coinciden en que:

[...] la administración de los tiempos es imprescindible para poder terminar los estudios, puesto que aparte de esas obligaciones se tienen el trabajo y la casa.

En relación a mención de la casa los varones expresaron "la atención a la familia" y las mujeres adicionaron la presencia en "las labores del hogar". Los informantes reiteran que si no fuera por la modalidad e-learning difícilmente pudieran ellos estar estudiando un posgrado. Gestionar los tiempos es vital para concluir desde una tarea, un trabajo, hasta toda su formación profesional.

- Control de las estrategias: es la acción que el estudiante realiza para administrar lo que está haciendo y evaluar si le está dando resultados favorables: $B, C$ y $D$ comentan:

[...] hay momentos en los que se tiene que checar si lo que se está haciendo es correcto.

Lo anterior refiriéndose a cualquier actividad que efectúan en la plataforma virtual. Los entrevistados manifiestan la importancia de saber si las acciones que realizan en la modalidad e-learning son las indicadas o no lo son, lo que hacen es modificarlas, cambiarlas y de ser necesario eliminarlas. Esto último, en parte, porque interfiere en 
su desempeño académico, por otro lado impacta de manera colateral en la optimización de los tiempos que en todos los casos dicen ser muy escaso.

Usos de la tecnología: proceso de organizar y unir las tecnologías que el alumno conoce, las cuales son adoptadas para su beneficio.

- Seleccionar de forma inteligente las tecnologías: acción para poder discernir, entre una amplia gama de tecnologías (videos, pdf, jpg, audios, u otro medio), cual es la más apropiada para sortear los requerimientos que se le imponen al alumno. $D$ y $F$ exponen que:

[...] es importante conocer todas la tecnologías y más si estás en la modalidad virtual de lo contrario estarías a la deriva, por eso es relevante conocer todas las tecnologías y si no por lo menos una buena parte.

- Determinar el momento de su utilización: establecer una sincronía entre la tecnología por usar $(\mathrm{T})$ y el momento para emplearla o requerirla (t) ni antes ni después, es decir, es la justa intersección entre $\mathrm{T}$ y t. En este sentido $A, C$ y $D$ aluden que:

[...] en ocasiones ocurre que cuando tienes tareas o ensayos por entregar hay que prestar atención cuál es la secuencia para poder realizar la actividades.

$B$ por ejemplo, indica:

[...] en la plataforma hay que ir bajando primero los PDF después hay que ver los videos, porque primero tienes que leer, para poder entender algún material audiovisual, al revés será muy complicado o no lo entenderías.

\section{Visión integradora del análisis de resultados empíricos obtenidos}

Para efectos de una mejor explicación a lo expresado, en la figura 3 se puede apreciar la relación que tiene la categoría núcleo con las categorías y subcategorías. Los resultados anteriores contribuyeron para elaborar la segunda Guía flexible con la cual se realizó otra ronda de entrevistas online como ya se hizo mención. Se precisa que la técnica de entrevista en profundidad y la MCC presentan similitudes en su proceder lo cual impacta de manera positiva y es coadyuvante para la investigación ya que permite trabajar el método y la técnica de manera paralela y/o simultánea. 
Figura 3. Categorías emergentes

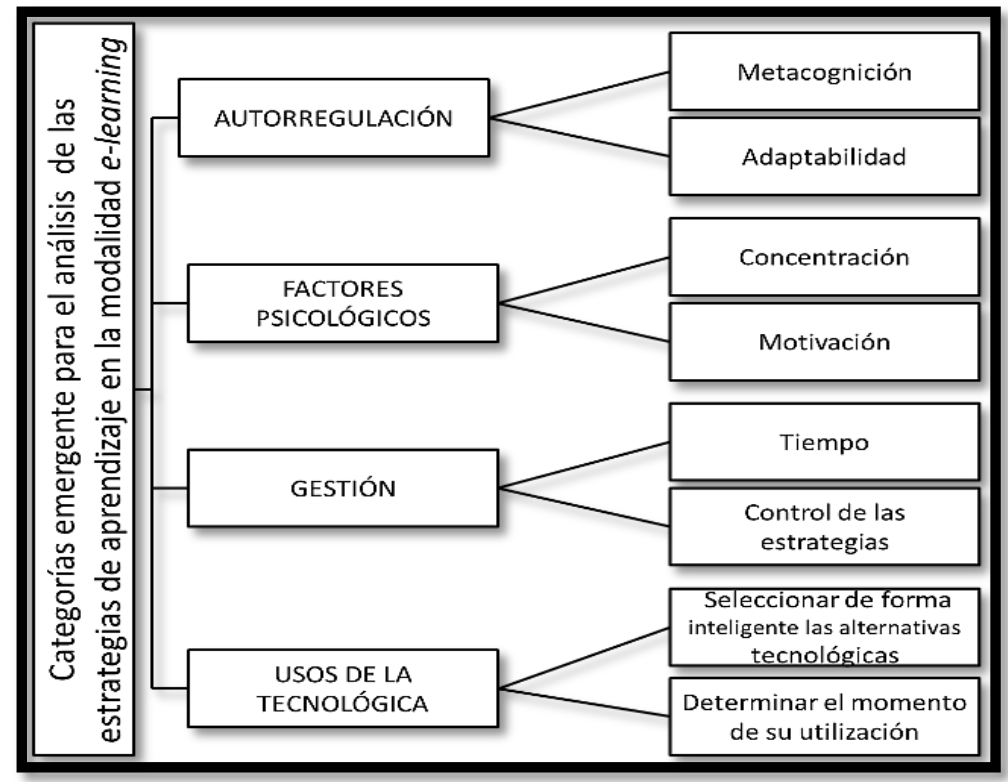

Fuente: elaboración propia.

Continuando con la fase del proceso de análisis de los datos apoyado en el software Atlas.ti, mediante la codificación axial y selectiva del MCC, permitió develar y hacer un reajuste, por lo que la categoría núcleo evoluciona a Dimensión de Estrategias de Aprendizaje en la Modalidad E-learning (DEAME), en este mismo sentido las llamadas categorías pasan ahora a ser denominadas factores, quedando de la siguiente manera: Factores Sicológicos (FS), Factores de Autorregulación (FA), Factores de Gestión (FG) y Factores de Uso de la Tecnología (FUT) y donde cada factor lo integran dos elementos, como se puede apreciar en la figura 4. De esta manera se puede tratar la temática que aquí acontece de un modo simplificado y ordenado para el caso de México pero que también se podrían ampliar al contexto latinoamericano con las reservas del caso.

Figura 4. Dimensión Estrategias de Aprendizaje en la Modalidad E-learning.

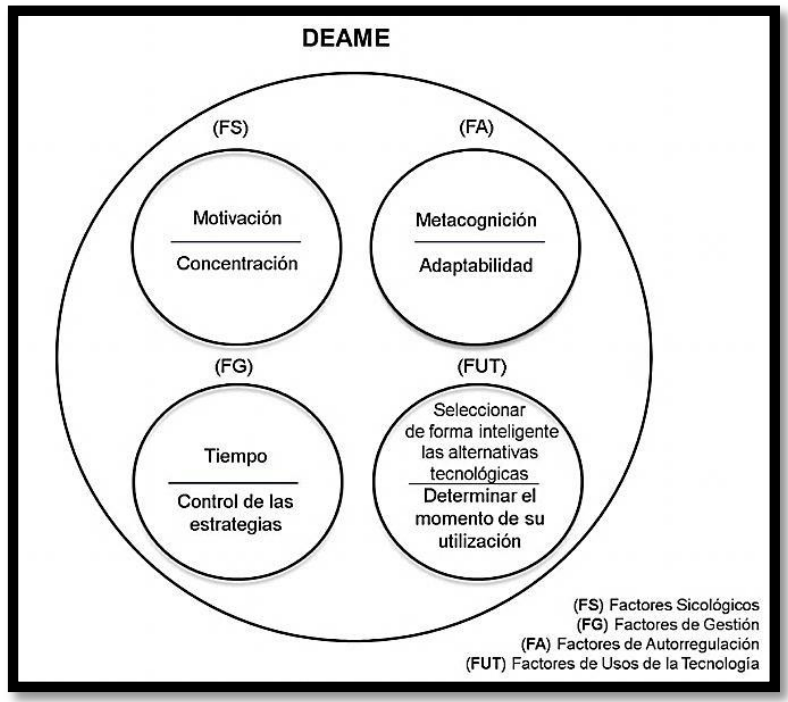

Fuente: elaboración propia.

Estrategias de aprendizaje emergentes en la modalidad e-learning. Luis Demetrio Meza-López, Ángel Serafín Torres-Velandia y José de Jesús Lara-Ruiz. 
Como parte de la investigación, también se indagó cuáles serían los elementos básicos para obtener un mejor aprendizaje en la modalidad e-learning con el uso de estrategias. De este análisis surge la figura 5, donde las líneas "punteadas" son las conexiones primarias o directas entre el factor y el código, y las líneas "continuas" son las que muestran conexión entre códigos de los distintos factores que no son primarias.

Figura 5. Relaciones básicas para lograr un aprendizaje con Estrategias de Aprendizaje en la Modalidad E-learning (EAME).

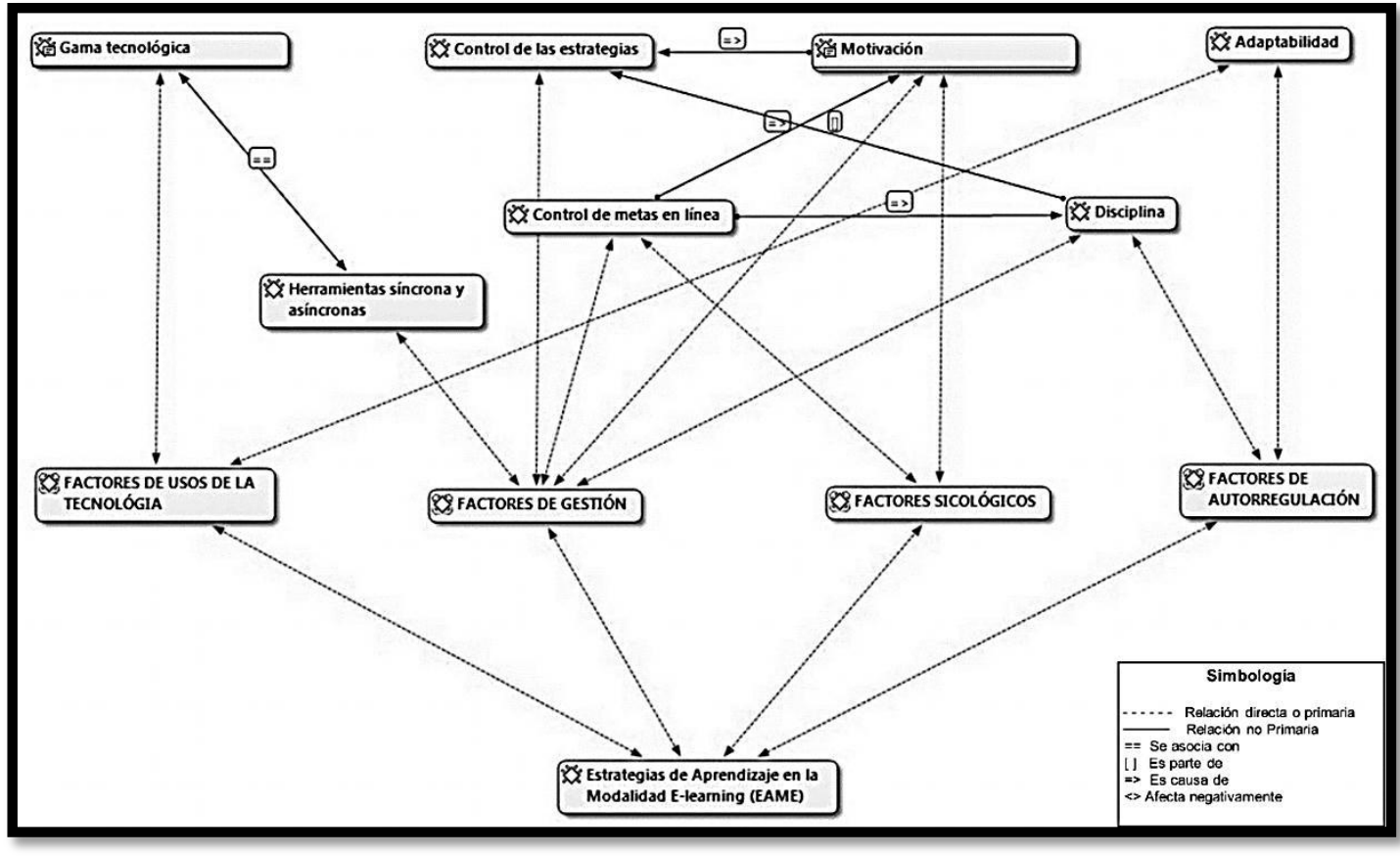

Fuente: elaboración propia.

Como se puede apreciar en la figura anterior, el Factor de Gestión es primordial para poder llevar a cabo el aprendizaje ya que este tiene injerencia en los otros tres factores, esto no quiere decir que por ello sea el más importante, lo que significa es que muy probablemente este factor es lo mínimo que un estudiante en el ámbito latinoamericano debe tomar en cuenta si pretende formase en la modalidad e-learning. Por lo que deberá saber gestionar las distintas Herramientas sincrónicas y asincrónicas logrando la selección de esa Gama tecnológica, así como tener un Control de las metas en línea, similarmente mantener la Motivación suficiente para seguir con sus estudios, todo esto amalgamado con la del código Disciplina que el estudiante va obteniendo en el trascurso de su formación.

Asimismo y como parte de la indagatoria se logró develar de manera emergente un entramado que agrupa a todos factores y todos los elementos con los cuales esta relacionado (familia de códigos en Atlas.ti) como se puede apreciar en la figura 6 y al igual que en la figura anterior las líneas "punteadas", que son las conexiones primarias o directas entre el factor y el código, así como las líneas "continuas" que son las que muestran conexiones no primarias. 
Figura 6. Red emergente para el análisis de las EAME (relación entre factores y elementos).

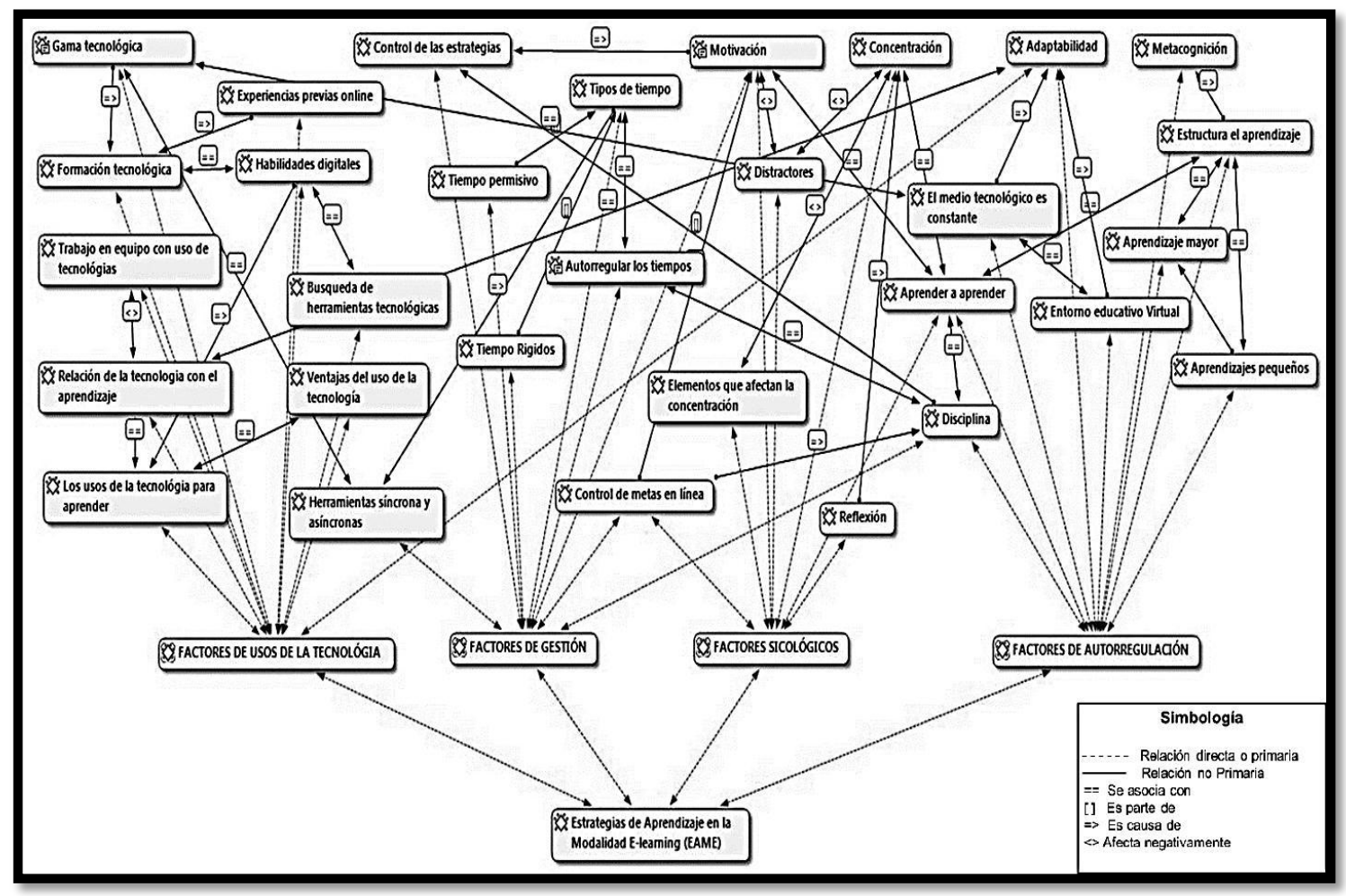

Fuente: elaboración propia.

La complejidad del entramado EAME (originado del corpus objeto de estudio) muestra para tres de los factores la coincidencia en el nombre de los elementos que la conforman y están relacionados con líneas "punteadas" como se observa en el caso de $F G$ (Control de estrategias y el Tiempo), FS (Motivación y Concentración), FA (Adaptabilidad y Metacognición), con excepción de FUT que para éste son implícitos. Asimismo se percibe la riqueza que esto representa al surgir elementos que posiblemente eran desconocidos. Ello representa develar elementos que no habían sido valorados ni tomados en cuenta para un estudio específico de las estrategias de aprendizaje en la modalidad e-learning, además la complejidad de relaciones entre factores puede ayudar a realizar una nueva propuesta de organización.

La validez y confiabilidad de los resultados concuerdan en la triangulación de los hallazgos obtenidos con el trabajo teórico de Tsai (2009) y Weinstein y Meyer (1998), en particular lo relacionado al factor de gestión y factores sicológicos ${ }^{9}$. Es decir se cumple el criterio de validez interna pero no el de validez externa según Guba y Lincoln (1989) Dado que se trata de un estudio de caso la perspectiva cualitativa no pretende generalizar los resultados o hallazgos de investigación a la manera de la estadística inferencial, dichos resultados se limitan al contexto de aplicación y para el caso de los sujetos participantes. Sin embargo, de acuerdo con la verificación de validez y confiabilidad tanto de los datos como de los resultados, es de esperarse que al

\footnotetext{
${ }^{9}$ En la validación de los resultados prioritariamente se tomó en cuenta la consistencia interna y sólo de manera complementaria la triangulación de los mismos con las teorías existentes (lo cual forma parte de las prácticas de la perspectiva cualitativa o interpretativa).
}

Estrategias de aprendizaje emergentes en la modalidad e-learning. Luis Demetrio Meza-López, Ángel Serafín Torres-Velandia y José de Jesús Lara-Ruiz. 
incrementar la muestra y su consideración en diversos contextos se encuentren resultados más o menos coincidentes con los matices correspondientes a la singularidad de los casos de estudio. Por lo anterior, se recomienda dar continuidad a la investigación tanto desde la perspectiva cualitativa como su consideración en un estudio de corte mixto (cualitativo y cuantitativo) ${ }^{10}$

Respecto al criterio de dependencia en cuanto a fiabilidad o consistencia de la investigación se cumple en tanto que las categorías emergentes surgieron de los datos mismos, aportados por los entrevistados. En cuanto a la confirmabilidad ligada a la neutralidad científica la posición fue de vigilar la objetividad de la subjetividad de los entrevistados respetando lo que es significativo e importante desde su cosmovisión. En suma los resultados empíricos obtenidos son concordantes con trabajos teóricos previos, lo que abona a la validez de constructo teórico a la par que el grado de saturación de las categorías favoreció la validez de contenido; con el agregado de que se destaca la diferenciación propia de la modalidad a distancia/virtual.

\section{Conclusiones}

Parte fundamental de la investigación fue develar la Dimensión Estrategias de Aprendizaje en la Modalidad E-learning (DEAME) y sus principales elementos, donde destacan la conceptualización de los siguientes factores:

- Factores de autorregulación, deben ser observados como la capacidad del estudiante para poder regular por sí mismo las acciones que sean necesarias para su aprendizaje. Donde la metacognición y adaptabilidad son elementos medulares para su conformación.

- Factores sicológicos, sobresalen la motivación y concentración, los cuales surgen de manera intrínseca en el alumno para favorecer el aprendizaje.

- Factores de gestión, en la modalidad e-learning estos factores son determinantes para que el alumno pueda aprender, pues es indispensable poder regular y/o controlar lo que acontece en la misma. Es de gran importancia el control del tiempo y la administración de las estrategias que el alumno va empleado.

- Factores de usos de la tecnología: proceso de organizar y unir las tecnologías que el alumno conoce, las cuales son adoptadas para su beneficio. Esto incluye que el alumno tenga la capacidad de poder seleccionar de forma inteligente las tecnologías así como determinar el momento de su utilización.

Asimismo se genera una primera aproximación a la conceptualización de las estrategias de aprendizaje en la modalidad e-learning. Se consideran como tales las acciones programadas por el alumno de manera cognitiva para aumentar su capacidad de aprender en dicha modalidad, donde el aprendiz es capaz de autorregular su

\footnotetext{
${ }^{10}$ Se hacela aclaración que este artículo es producto de un trabajo de investigación de tres años con dedicación de tiempo completo, de manera que pretender hacer un trabajo riguroso de carácter científico para el contexto de Latinoamérica al menos haría necesario dedicar un tiempo similar, lo cual más que un proyecto sería un programa de investigación como lo define Lakatos (1983).
}

Estrategias de aprendizaje emergentes en la modalidad e-learning. Luis Demetrio Meza-López, Ángel Serafín Torres-Velandia y José de Jesús Lara-Ruiz. 
metacognición y de adaptarse a situaciones emergentes propias del medio; además, factores sicológicos como la motivación y la concentración son determinantes para emprender cualquier acción estratégica; de igual manera lo es el poder gestionar las actividades a realizar, así como determinar los tiempos para su aplicación en la modalidad virtual. Resulta de relevancia que el discente sea apto para discernir y determinar el uso de las tecnologías disponibles en la modalidad, presuponiendo que las conoce y sabe cómo funcionan para conseguir la mayor utilidad. Por lo que el estudiante tiene el control de las acciones que va implementando y por ello puede decidir si le funcionan o no, si funcionan continua con las estrategias, si no las adecua o las cambia. Precisando que algunos alumnos emplean algunas o todas las estrategias posibles en la modalidad e-learning, lo cual está determinado en función del grado de habilidad de las estrategias que posee o es capaz de desarrollar.

Finalmente, se sugiere la búsqueda de nuevas categorías emergentes y profundizar en las relaciones entre las mismas, también el de retomar los hallazgos develados para evaluar las estrategias de aprendizaje en la modalidad e-learning. En esto radica parte de la relevancia de la investigación ya que se logran hacer algunas contribuciones novedosas en la forma que los estudiantes toman o adecuan las estrategias de aprendizaje en la educación virtual en México y que estas mismas pudieran ser encontradas y estudiadas en el contexto latinoamericano.

Presentación del artículo: 29 de noviembre de 2015 Fecha de aprobación: 14 de diciembre de 2015 Fecha de publicación: 30 de enero de 2016

Meza-López, L.D., Torres-Velandia, A.S. y Lara-Ruiz, J.de J. (2016). Estrategias de aprendizaje emergentes en la modalidad e-learning. RED. Revista de Educación a Distancia. 48(5). Consultado el (dd/mm/aaaa) en http://www.um.es/ead/red/48/meza.pdf

\section{Bibliografía}

Arroyo, M. \& Miklos, T; (2008). Una visión prospectiva de la educación a distancia en América Latina. Universidades, LVIII (37) 49-67. Recuperado el 11 de noviembre de 2015 de: http://redalyc.org/articulo.oa?id=37311274005

Berger, P. \& Luckmann, T. (1993). La construcción social de la realidad, Buenos Aires: Amorrortu.

Blumer, H. (1969). Symbolic interactionism: Perspective and Method. New Jersey: Prentice-Hall.

Boneu, Josep M. (2007). Plataformas abiertas de e-learning para el soporte de contenidos educativos. Recuperado y consultado el 26 de febrero de 2015 de: http://www.uoc.edu/rusc/4/1/dt/esp/boneu.pdf 
Collis, B. \& Meeuwsen, E. (1999).Learning to learn in a WWW-based environment. In French, D., Hale, C., Johnson, C. \& Farr, G. (Eds.), Internet Based Learning-A Framework for Higher Education and Business. Sterling: Stylus Publishing, pp. 2546.

Derry, S. J. \& Murphy, D, (1986). Designing systems that train learning ability: from theory to practice. Review of educational research, 56, 1-39.

Flick, U. (2007). Introducción a la investigación cualitativa. Madrid: Morata.

Glaser, B. \& Strauss, A. (1967). The discovery of grounded theory: strategies for qualitative research, Chicago: Aldine de Gruyter.

Gros-Salvat, Begoña. (2011). Evolución y retos de la educación virtual. Construyendo el e-learning del siglo XXI. Barcelona: Editorial UOC.

Guba, Egon G. \& Lincoln, Yvonne S. (1989). Fourth generation evaluation. Newbury Park: Sage.

Lakatos, I. (1983). La metodología de los programas de investigación científica. Ed. cast.; Madrid, Alianza Editorial.

Marcelo, C., M.A. Ballesteros \& A. Palazón. (2002). e-learning teleformación. Diseño, desarrollo y evaluación de la formación a través de Internet. Barcelona: Gestión 2000.

Mayor, J., Suengas,A. \& González-Marqués, J. (1995). Estrategias Metacognitivas: Aprender a aprender y aprender a pensar. Barcelona: Síntesis.

Mead, G. (1964). George Herbert Mead on social psychology. Chicago: University of Chicago Press.

Milkos, T. \& Arroyo, M. (Coord.). (2008). El futuro de la educación a distancia y del elearning en América Latina. Una visión prospectiva. México: ILCE.

Pérez, A. et al. (2003). Estrés y rendimiento académico en estudiantes universitarios. Medicina Psicosomática y Psiquiatría de Enlace, 67, 26-33. Recuperado el 12 de febrero de 2012 de: http://search.ebscohost.com.

Pintrich, P. \& García, T. (1993). Intraindividual differences in students' motivation and self-regulated learning. German Journal of Educational Psichology, vol.7 (3), 99107.

Pintrich, P. et al. (1991). Manual for the use of the Motivated Strategies for Learning Questionnaire (MSLQ).The University of Michigan, Ann Arbor, MI. 
Robles, B. (2011). La entrevista en profundidad: una técnica útil dentro del campo antropofísico. Cuicuilco, [en línea] 2011, 18 (Septiembre-Diciembre). ISSN 14057778. Recuperado y consultado el 11 de marzo de 2015 de: http://www.redalyc.org/articulo.oa?id=35124304004

San Martín, D. (2014). Teoría fundamentada y Atlas.ti: recursos metodológicos para la investigación educativa. Revista Electrónica de Investigación Educativa, 16(1), 104122. Recuperado y consultado el 18 de abril de 2015 de: http://redie.uabc.mx/vol16no1/contenido-sanmartin.html

Strauss, A. \& Corbin, J. (2002). Bases de la investigación cualitativa. Técnicas y procedimientos para desarrollar la Teoría Fundamentada. Colombia: Universidad de Antioquia.

Tsai, M.J. (2009). The Model of Strategic e-Learning: Understanding and Evaluating Student e-Learning from Metacognitive Perspectives. Educational Technology \& Society, 12 (1), 34-48. Recuperado y consultado el 21 de marzo de 2015 de: http://www.springerlink.com.

Weinstein, C. \& Mayer, R. (1986). The teaching of learning strategies. En M.C. Wittrock (Ed.), Handbook of research on teaching. New York: Collier Macmillan

Weinstein, C. E. \& Meyer, D.K. (1998). Implicaciones de la psicología cognitiva en la aplicación de pruebas: contribuciones a partir del trabajo realizado en estrategias de aprendizaje. En Wittrock, M. \& Baker, E.L. (Comp.). Test de cognición: Investigación cognitiva y mejora de las pruebas psicológicas. Barcelona, Paidós, pp.65-92.

Weitzman, E. \& Miles, M. (1995).Computer programs for qualitative data analysis. London: Sage.

Zambrano, W.R. \& Medina, V. H. (2010). Creación, implementación y validación de un modelo de aprendizaje virtual para la educación superior en tecnología Web 2.0., Signo y pensamiento, 56. Documentos de investigación, volumen XXIX, pp. 288303. Recuperado y consultado el 15 de octubre de 2015 de:

http://revistas.javeriana.edu.co/index.php/signoypensamiento/article/view/2564/1833 